\title{
Review
}

\section{Urinary Bladder Carcinogenesis by DNA Reactive and Non-Reactive Chemicals: Non-Linearities and Thresholds}

\author{
Samuel M. Cohen ${ }^{1}$ \\ Department of Pathology and Microbiology and Eppley Cancer Center, University of Nebraska Medical Center, NE, USA
}

(Received July 28, 2012; Revised August 21, 2012; Accepted August 22, 2012)

\begin{abstract}
Chemicals can increase the risk of cancer by either directly damaging DNA (DNA reactive) or increasing cell proliferation. DNA reactive carcinogens involve activation to reactive metabolites, forming DNA adducts which are mutagenic. The presence of numerous cellular repair processes suggest that these could have a threshold. The issues involved are described for 2-acetylaminofluorene urinary bladder carcinogenicity. Chemicals that act by increasing cell proliferation involve either increased cell births or decreased cell deaths, leading to an accumulation of cells. Multiple mechanisms can produce these effects, most of which have threshold processes. Arsenicals appear to act by inducing cellular cytotoxicity with regenerative proliferation, induced by generation of reactive trivalent forms which interact with critical sulfhydryl groups in cells. A more definitive threshold response is illustrated for the formation of urinary solids, either calculi (melamine) amorphous calcium phosphate-containing precipitate (sodium saccharin) or crystalluria (PPAR $\gamma$ agonists). Increasing evidence strongly supports the concept of thresholds in carcinogenesis, not only for chemicals acting by increasing cell proliferation but also for those acting by DNA reactivity.
\end{abstract}

Key words: urinary bladder, acetylaminofluorene, arsenic, melamine, saccharin, PPAR $\gamma$ agonists

\section{Introduction}

The issue of thresholds in chemical carcinogenesis has pervaded the field of carcinogenesis for more than seven decades. It arose primarily by the observation that DNA reactive carcinogens behaved much like radiation (1). Since radiation appeared to have a non-threshold response for cancer induction, it was assumed that a similar process could occur with chemicals. Many of the known carcinogens of the 1950s and 1960s were demonstrated to be metabolically activated to metabolites that reacted covalently with DNA leading to the formation of DNA adducts, many of which were mutagenic (2), providing strong support for a closer association with radiation, experimentally and theoretically.

However, the concept of linearity with radiation-induced carcinogenesis has recently been questioned (3).
Likewise, the likelihood that carcinogenesis, even for DNA reactive carcinogens, could have a threshold has also become a topic of considerable interest and concern. In addressing the concept of thresholds in carcinogenesis, it is critical to separate the processes that have been identified for DNA reactive carcinogens compared to those chemicals that produce cancer without forming DNA reactive metabolites or DNA adducts $(4,5)$.

\section{Terminology}

In discussions regarding thresholds, unfortunately, there has been confusion in terminology by interchangeably using several other terms to imply thresholds. The separation of these terms and their distinct meanings is critical to any discussion regarding thresholds. To begin with, the term non-linearity is not the same as thresholds. Non-linearities can occur for a variety of reasons, saturation of metabolic processes, competing dose responses, hormetic effects, and others. Although in some of these cases the non-linearity will actually have a true threshold, these terms need to be distinguished. The more difficult distinction is between a no observed effect level (NOEL) compared to the term threshold. With increasing technology, our ability to detect various responses has increased, so that levels that were once thought to be thresholds have turned out not to be, since the level of detection was significantly improved. This has become particularly true given the incredible developments in analytical chemistry during the past few decades.

For example, aflatoxin is a known liver carcinogen for many species, including humans $(6,7)$. Initially, there was the possibility of a threshold response since low levels of the chemical could not be detected. With advances in analytical chemistry, we can now detect aflatoxin to levels as low $10^{-18}$, implying that aflatoxin is present in all peanut products, albeit at extremely low levels. Fur-

${ }^{1}$ Correspondence to: Samuel M. Cohen, Department of Pathology and Microbiology and Eppley Cancer Center, University of Nebraska Medical Center, Omaha, NE 68198-3135, USA. Tel: +1-402559-6388, Fax: + 1-402-559-8330, E-mail: scohen@unmc.edu doi: $10.3123 /$ jemsge. 34.165 
thermore, the detection of a biologic response is generally much less sensitive than analytical chemistry. Cancer detection, as well as detection of prenoplastic lesions such as hepatocellular foci, is particularly not sensitive (8). Thus, the observation of a no observed increase in tumors at a given dose does not necessarily imply a true threshold. For an experiment with only 50 animals per group, the level of detection is an increase above the spontaneous level of approximately 10 percent. Obviously, such a high incidence would be intolerable in a human population, where extrapolations are generally to levels of lifetime increased risk of cancer of 1 in 100,000 or 1 in $1,000,000$. To obtain such extrapolations, theoretical models have to be used. This is where the issue of no observed effect levels compared to true thresholds becomes an issue (8-10). It is essential when conceptualizing the extrapolation to lower doses that the model is biologic rather than statistical. Frequently, extrapolation from observed response levels to levels below which an observable response can be detected will fit a variety of mathematical models, some of which have thresholds and some of which do not. Only biological understanding can be used to make a realistic extrapolation to the low dose.

\section{Carcinogenesis}

Cancer is a complex process, but our understanding has advanced considerably (4). We now know that cancer is due to multiple genetic effects occurring in a single target cell, giving rise to the clonal origin of cancers. It is clear that more than one genetic alteration is required, although the actual number is unknown. Furthermore, we know that every time DNA replicates, mistakes occur, albeit infrequently. The mutation rate for DNA replications in somatic cells varies with cell types, with frequent mutations occurring in lymphoid cells whereas mutations occur much less frequently in many epithelial cell systems. Nevertheless, every time a cell replicates, there is a chance for mistakes. It is only when all of the critical changes in DNA occur in a single cell that cancer ultimately develops.

Based on these observations, there are ultimately only two major ways that a chemical can increase the risk of cancer: 1) the chemical can directly damage DNA, leading to an increase in the probability that a critical mistake leading to cancer will occur each time DNA replicates; or 2) the number of DNA replications can be increased, increasing the probability of spontaneous mistakes occurring. Since DNA replications are occurring in most tissue systems, there is a background incidence of cancer risk. It is only when the number of DNA replications or the number of mistakes per replication are increased that there is an increased risk for developing cancer $(4,9,11)$.

There are numerous pathways that have been de- scribed for metabolic activation of chemicals leading to DNA reactive metabolites $(2,12)$. Ultimately, the effect is to react with the DNA forming an adduct that is mutagenic. Just having DNA adducts does not necessarily mean that they are mutagenic, as some DNA adducts do not lead to mutations. Furthermore, any time an adduct forms, there are numerous repair processes which eliminate the possibility for mutation. These vary considerably by the type of adduct that is formed, particularly with differences between small versus large, bulky adducts (4).

Likewise, increases in cell proliferation can occur by several mechanisms (4). Fundamentally, however, they occur either by increasing cell births or decreasing cell deaths. Increasing cell births can occur either by direct mitogenesis, usually associated with hormones or growth factors, or by toxicity, cell death, and regenerative proliferation. Decreases in cell death can occur either by inhibiting apoptosis or inhibiting differenation, which is a cell death process. By inhibiting cell death, there is an accumulation of cells. Even if the rate of DNA replication remains the same as in controls, the number of DNA replications, the critical parameter, will be increased.

It is essential that the mistakes that are induced in the DNA occur in a single cell, since cancer is a clonal process, and it is essential that the cell in which these mistakes occur is part of the pluripotential (stem) cell population of a tissue (4). Cells that are committed to differentiation or fully differentiated are essentially committed to dying. These will not evolve into cancer cells, since the continuity for cell lineage is essential.

Based on these concepts, it is evident that the critical parameters for each step in the process of developing cancer are fundamentally the probability that each time DNA replicates a critical mistake will occur in one of the genes leading to cancer and the number of DNA replications. The number of DNA replications is dependent on the cell number and the rate at which replication is occurring. Each of these parameters can be assessed, and if any one of them is increased, there is a potential increase in the risk of developing cancer.

\section{DNA Reactive Carcinogens}

I use the term DNA reactive specifically rather than the broader term of genotoxicity (5). Genotoxicity includes alterations in DNA due to DNA adduct formation and mutations, but also includes other genetic processes such as aneuploidy, translocations, deletions, and other genetic alterations. However, the other genotoxic endpoints beside specific DNA reactivity are generally indirect processes, secondary to alternations in cellular processes that indirectly affect DNA (13). For these genotoxic, but non-DNA reactive processes, there is strong theoretical and experimental evidence that there 
can be a threshold involved. The ultimate question comes to the possibility of a threshold for DNA-reactive carcinogens. The difficulty in addressing the possibility of a threshold for such agents, is our very limited abilities for detecting the biological phenomena, such as mutation, preneoplastic and neoplastic formation. Nevertheless, on purely theoretical grounds, the recent arguments being developed for possible thresholds for radiation-induced mutation and carcinogenesis can be applied to DNA-reactive carcinogens (14).

Detection of such agents relies primarily on a variety of methods, including structure activity relationships, the Ames mutation assay in Salmonella, and specific assays for detection of DNA adducts, such as ${ }^{32} \mathrm{P}$ postlabeling (5).

\section{Acetylaminofluorene}

The issues concerned in the dose response assessment for DNA-reactive carcinogens was illustrated well by the large study performed in the 1970s evaluating 2acetylaminofluorene (AAF) administered to female mice, with liver and urinary bladder tumors being induced $(8,15)$. Large numbers of animals (greater than 24,000 ) were used so that the level of detection would be an increased incidence of $1 \%$ in contrast to the usual $10 \%$ detectability in a standard two-year bioassay. Interim sacrifices were performed at 18 and 24 months with a terminal sacrifice at 33 months. Furthermore, stop feeding experiments were included, with the agent being administered in the diet. Low doses were used, ranging from 30 to $150 \mathrm{ppm}$ of the diet.

The dose response for liver tumors was nearly linear, whereas the dose response for the urinary bladder tumors was clearly sigmoidal, with a significant increase in bladder tumor incidence only seen at $60 \mathrm{ppm}$ and above.

However, evaluation of DNA adducts in the bladder and liver tissues showed that there were actually more AAF DNA adducts in the bladder per unit of DNA compared to the liver, but more importantly, the dose response for DNA adducts was linear in both the bladder and liver down to doses considerably lower than those used in the carcinogenicity study $(8,16)$. Again, this is an example of level of detection being of paramount importance, since detection limits for DNA adducts are considerably more sensitive than tumor formation.

The reason for the increase in tumor incidence at 60 ppm and above in the bladder was because this was the dose at which there was not only DNA adduct formation, but an increase in cell proliferation in the urothelium. The combination of increased DNA adducts and increased cell numbers proliferating at a higher rate than normal provided an adequate milieu for development of a detectable incidence of tumors. However, modeling of the process, taking into account DNA replication and DNA adduct formation, indicates that there would also be an increased incidence of tumors at lower doses, below the level of detection (1\%) for tumors. The incidences would be expected to be less than $0.1 \%$, well below the level of detection for tumors, but not for DNA adducts $(8,15,16)$. This is an example of nonlinearity, the issue of threshold being complicated by issues related to level of detection.

\section{Arsenic Carcinogenesis}

Although evidence is accumulating that DNA adduct formation and specific mutagenesis may actually have a threshold $(3,14)$, this remains a controversial issue. As more definitive data are developed, this issue can be resolved. However, it will be reliant on development of technologies that are highly sensitive so that level of detection does not become an issue.

Inorganic arsenic is a known human carcinogen, inducing tumors of the skin, lung, and urinary bladder (17). Although there is evidence, primarily in vitro, that it can produce genotoxic effects, this occurs at high concentrations and by indirect mechanisms (18). Arsenicals do not interact directly with DNA, so it is not DNA reactive. Instead, it would appear that inorganic arsenic and a methylated arsenical, dimethyl arsinic acid (DMA) cause cytotoxicity in the target tissue, leading to regenerative proliferation which is sustained over time resulting eventually in the development of tumors (17-19). It remains controversial, but the evidence is accumulating that arsenic carcinogenesis and toxicity are threshold phenomena.

This has been best studied utilizing the example of DMA induction of bladder tumors in the rat (18). DMA is negative in the two-year bioassay in mice. DMA in rats rapidly induces cytotoxicity of the urothelium that is evident by scanning electron microscopy, with consequent increased cell proliferation due to regeneration. Arsenicals are known to be highly reactive in the trivalent form, and for DMA this is also true. DMA is administered in the pentavalent form, but is metabolized to the trivalent form and excreted in the urine at concentrations that are adequate to produce the cytotoxicity, based on in vitro and in vivo investigations (18). DMA is further methylated to trimethylarsenic oxide (TMAO), which is also pentavalent and relatively non-toxic. In addition to the trivalent DMA ${ }^{\mathrm{III}}$, DMA also is metabolized to thiolated metabolites, some of which are highly cytotoxic. These are cytotoxic because of their ability to rapidly enter the cell and then rapidly be converted from the pentavalent thiolated arsenical to the highly reactive, trivalent DMA ${ }^{\mathrm{III}}$.

There is a clear dose response for DMA in the rat, with no evidence of changes present in the urothelium at an oral dose of $2 \mathrm{ppm}$ DMA, whereas detectable 
changes are seen at doses of $10 \mathrm{ppm}$ and higher, with tumorigenicity occurring at $100 \mathrm{ppm}$ of the diet (18). This has also been corroborated in studies administering the DMA in the drinking water. Of critical importance is the observation that potentially cytotoxic concentrations of DMA ${ }^{\mathrm{III}}$ are excreted in the urine of the rats that are administered $\mathrm{DMA}^{\mathrm{v}}$ in the diet or drinking water at concentrations of $10 \mathrm{ppm}$ and above, but they are not detected in the rats administered the $\mathrm{DMA}^{\mathrm{v}}$ as $2 \mathrm{ppm}$ of the diet. Clearly, this provides a biological basis for a threshold, which is dependent on an adequate concentration of the metabolites being excreted in the urine that is sufficient for producing the cytotoxicity. If the concentration of the critical metabolite (DMA ${ }^{\mathrm{III}}$ ) is below this threshold, then there is not a cellular response, such as cytotoxicity, regeneration, and tumors. Thus, the evidence strongly supports the concept of a threshold for arsenic-induced carcinogenesis in the rat.

There is also accumulating evidence in humans that inorganic arsenic, which has a mode of action similar to DMA, also has a threshold for carcinogenesis in humans based on epidemiology studies (20). A study by Lamm et al. (21) reexamined the Taiwanese data based on precinct and showed a threshold at approximately $150 \mathrm{ppb}$ of exposure in the drinking water. Several recent epidemiology studies of populations exposed to relatively low levels of inorganic arsenic (less than 100 $\mathrm{ppb}$ ) in the drinking water also give negative results with respect to tumorigenicity (20). Most of these studies have been related to the bladder, but there is similar evidence accumulating for the lung and skin. Such conclusions are supported not only by epidemiology investigations, which are relative crude measures, but also are based on sound biological principles, including the necessity for the presence of an adequate concentration of the trivalent arsenical in the target tissue that is sufficient for inducing cytotoxicity, or for that matter, any biological response.

\section{Urinary Calculi}

The clearest evidence for thresholds in carcinogenesis involves the mode of action involving production of urinary calculi and associated urinary bladder neoplasms (22-24). This has been best investigated in the rat, but also has been identified in the mouse. Its relevance to humans remains controversial, since calculi are rarely present in humans for a sustained period of time (they cause obstruction and excruciating pain and removed after a short period of time) $(24,25)$. In the unusual circumstances in which they are present for long periods of time (diverticuli, neurogenic bladder) bacterial cystitis is also present, a known risk factor for bladder cancer in humans. In animal models, development of urinary tract calculi is dependent entirely on the solubility of the agent involved. Only when a concentration that exceeds the solubility of the chemical do calculi form (22-26). This is dependent on a definable physical property of the chemical, a true physical chemical threshold related to a true biological threshold. Once calculi form in the urinary tract, they act as an abrasive leading to urothelial toxicity, frequently ulceration, and consequent marked regenerative proliferative generation which continues as long as the calculus is present. The extent of the proliferation is enormous, with an increase in labeling index of more than 100 times compared to controls and an increase in cell number of more than 1,000 times. This results in a replication number that is more than 5 orders of magnitude greater than controls. It is not surprising that a marked increase in bladder tumors frequently is associated with such stimuli. The induction of the tumors is a consequence of the increased proliferation which is a consequence of the cytotoxicity, which is a consequence of the presence of urinary tract calculi, which is a consequence of the precipitation of a material because it has exceeded a threshold level related to its solubility. The carcinogenic agent is the calculi, not the chemical itself.

Numerous substances have been identified that produce urinary calculi, but the concept of threshold was particularly evident in a recent unfortunate example involving melamine (27). Melamine is present in the environment because of its use in plastics and in agriculture, but at exceedingly low levels, usually less than a microgram per day per person (26). However, at high concentrations it had been shown in rats and mice that urinary tract calculi could be produced by melamine administration at high doses, and in rats, there was an increased incidence of bladder tumors secondary to the calculi, not to the chemical melamine itself. However, the exposures required for calculus development in the rodents was approximately 5 orders of magnitude greater than human exposures, thus no evidence of toxicity or carcinogenicity was expected in the human population from this chemical (26).

However, a few years ago there was an episode of contamination of infant formula with extremely high levels of melamine, leading to the induction of urinary tract calculi in these infants (27). Fortunately, treatment could be performed to correct this problem in most of the infants, without known sequelae. Nevertheless, the amount of melamine was certainly sufficient to produce urinary tract calculi, having far exceeded the threshold level necessary for precipitation in the urine. There is no information regarding the ultimate induction of tumors, but given the transient nature of the calculi, it is unlikely that there was carcinogenicity. However, this is clear evidence of a threshold involved in toxicity and, at least in the animal models, a clear threshold for carcinogenicity. 


\section{Urinary Solids}

An indirect process leading to urinary tract calculi also involves a threshold and has been illustrated with examples including sodium salts, such as sodium saccharin and sodium ascorbate, which in rats leads to excess amounts of calcium phosphate being present in the urine leading to precipitation, urothelial proliferation, and ultimately a low incidence of bladder tumors. This is a rat specific phenomenon, but clearly involves a threshold $(28,29)$.

A similar process was identified with PPAR $\gamma$ and dual $\operatorname{PPAR} \gamma / \operatorname{PPAR} \alpha$ agonists in rats $(25,30)$. Mechanistically this was shown to involve inhibition of citrate synthesis by the PPAR $\gamma$ agonists leading to hypocitratemia with consequent hypocitraturia. Since citrate is the major chelating substance in urine keeping calcium in solution, the significant lowering of citrate led to precipitation of calcium-containing urinary solids, including calculi. This led to the typical sequence of toxicity, necrosis, regenerative proliferation, hyperplasia and ultimately tumors. Again, this involves a process that is clearly a threshold response related to the physical chemical properties of calcium and phosphate, indirectly influenced by the PPAR $\gamma$ agonists.

\section{Conclusions}

There is extensive evidence that non-DNA reactive carcinogens, such as sodium saccharin, arsenic, and PPAR $\gamma$ agonists have a threshold mode of action for development of toxicity and tumors. For DNA reactive carcinogens, such as AAF, the question remains unclear, although accumulating evidence suggests that there is a threshold involved in this process. This will be greatly influenced by the potential for threshold mechanisms in the metabolic activation versus inactivation as well as the ability for cells to repair DNA adducts that are formed. Ultimately, the conclusion as to whether a threshold is present or not must rely on a theoretical model that includes threshold phenomena since demonstration of a true threshold in laboratory or epidemiology studies will be difficult if not impossible because of the issue of level of detection.

\section{References}

1 U.S. National Academy of Sciences. Biological effects of atomic radiation. Washington, DC: 1956.

2 Miller JA, Miller EC. Ultimate chemical carcinogens as reactive mutagenic electrophiles. In: Hiatt, editor. Origins of Human Cancer. Cold Spring Harbor Laboratory, Cold Spring Harbor; 1977. p. 605-27.

3 Calabrese EJ. Key studies used to support cancer risk assessment questioned. Environ Mol Mutagen. 2011; 52: 595-606.

4 Cohen SM, Ellwein LB. Genetic errors, cell proliferation, and carcinogenesis. Cancer Res. 1991; 51: 6493-505.
5 Cohen SM, Arnold LL. Chemical carcinogenesis. Toxicol Sci. 2011; 120: 76-92.

6 Kensler TW, Roebuck BD, Wogan GN, Groopman JD. Aflatoxin: a 50-year odyssey of mechanistic and translational toxicology. Toxicol Sci. 2011; 120 Suppl 1: S28-48.

7 Chu FS. Mycotoxins: food contamination, mechanism, carcinogenic potential and preventive measures. Mutat Res. 1991; 259: 291-306.

8 Cohen SM, Ellwein LB. Proliferative and genotoxic cellular effects in 2-acetylaminofluorene bladder and liver carcinogenesis: biological modeling of the $\mathrm{ED}_{01}$ study. Toxicol Appl Pharmacol. 1990; 104: 79-93.

9 Moolgavkar SH, Knudson AG Jr. Mutation and cancer: a model for human carcinogenesis. J Natl Cancer Inst. 1981; 66: 1037-52.

10 Crump KS. Use of threshold and mode of action in risk assessment. Crit Rev Toxicol. 2011; 41: 637-50.

11 Greenfield RE, Ellwein LB, Cohen SM. A general probabilistic model of carcinogenesis: analysis of experimental urinary bladder cancer. Carcinogenesis. 1984; 5: 437-45.

12 Parkinson A, Ogilvie BW. Biotransformation of xenobiotics. In: Klaassen C. editor. Toxicology the Basic Science of Poisons. New York: The McGraw-Hill Companies, Inc.; 2008. p. 161-304.

13 Zeiger E. History and rationale of genetic toxicity testing: an impersonal, and sometimes personal, view. Environ Mol Mutagen. 2004; 44: 363-71.

14 Gocke E, Muller L. In vivo studies in the mouse to define a threshold for the genotoxicity of EMS and ENU. Mutat Res. 2009; 678: 101-7.

15 Farmer JH, Kodell RL, Greenman DL, Shaw GW. Dose and time response models for the incidence of bladder and liver neoplasms in mice fed 2-acetylaminofluorene continuously. J Environ Pathol Toxicol. 1979; 3: 55-68.

16 Beland FA, Fullerton NF, Kinouchi T, Smith BA, Poirier MC. DNA adduct formation in relation to tumorigenesis in mice chronically fed 2-acetylaminofluorene. Prog Clin Biol Res. 1990; 331: 121-9.

17 Cohen SM, Ohnishi T, Arnold LL, Le XC. Arsenic-induced bladder cancer in an animal model. Toxicol App Pharmacol. 2007; 222: 258-63.

18 Cohen SM, Arnold LL, Eldan M, Schoen AS, Beck BD. Methylated arsenicals: the implications of metabolism and carcinogenicity studies in rodents to human risk assessment. Crit Rev Toxicol. 2006; 36: 99-133.

19 Suzuki S, Arnold LL, Ohnishi T, Cohen SM. Effects of inorganic arsenic on the rat and mouse urinary bladder. Toxicol Sci. 2008; 106: 350-63.

20 Mink PJ, Alexander DD, Barraj LM, Kelsh MA, Tsuji JS. Low-level arsenic exposure in drinking water and bladder cancer: a review and meta-analysis. Regul Toxicol Pharmacol. 2008; 52: 299-310.

21 Lamm SH, Engel A, Penn CA, Chen R, Feinleib M. Arsenic cancer risk confounder in southwest Taiwan data set. Environ Health Perspect. 2006; 114: 1077-82.

22 Cohen SM, Johansson SL, Arnold LL, Lawson TA. Urinary tract calculi and thresholds in carcinogenesis. Food Chem Toxicol. 2002; 40: 793-99. 
23 Clayson DB, Fishbei, L, Cohen SM. The effect of stones and other physical factors on the induction of rodent bladder cancer. Food Chem Toxicol. 1995; 33: 771-84.

24 IARC Working Group. Consensus Report. International Agency for Research on Cancer, IARC Scientific Publications, 1999; 147: 1-32.

25 Dominick MA, White MR, Sanderson T, Van Vleet TR, Cohen SM, Arnold LL, Cano M, Tannehill-Gregg S, Moehlenkamp JD, Waites CR, Schilling BE. Urothelial carcinogenesis in the urinary bladder of male rats treated with muraglitazar, a PPAR $\alpha / \gamma$ agonist: evidence for urolithiasis as the inciting event in the mode of action. Toxicol Pathol. 2006; 34: 903-20.

26 Meek ME, Bucher JR, Cohen SM, Dellarco V, Hill RN, Lehman-McKeeman LD, Longfellow DG, Pastoor T,
Seed J, Patton DE. A framework for human relevance analysis of information on carcinogenic modes of action. Crit Rev Toxicol. 2003; 33: 591-653.

27 Wang IJ, Chen PC, KC Huang. Melamine and nephrolithiasis in children in Taiwan. $\mathrm{N}$ Engl $\mathrm{J}$ Med. 2009; 360: 1157-8.

28 Cohen SM. Calcium phosphate-containing urinary precipitate in rat urinary bladder carcinogenesis. International Agency for Research on Cancer, IARC Scientific Publications. 1999; 147: 175-89.

29 Cohen SM. Urinary bladder carcinogenesis. Toxicol Pathol. 1998; 26: 121-7.

30 Cohen SM. Effects of PPAR $\gamma$ and combined agonists on the urinary tract of rats and other species. Toxicol Sci. 2005; 87: 322-7. 